\title{
A Product-Coded WDM Coding System
}

\author{
Ming-Seng Kao, Member, IEEE, Hsin-Yuan Chen, and Das Lin
}

\begin{abstract}
Forward error correction is a feasible approach to decline the bit error floor of lightwave systems, arising from the fact that a simple single-error-correcting code can reduce the error floor from $O\left(P_{e}\right)$ to $O\left(P_{e}^{z}\right)$. Here we propose a novel wavelength demultiplexer (WDM) coding system using product code to improve performances of WDM systems with an error floor caused by fiber dispersion or system noises.
\end{abstract}

\section{INTRODUCTION}

$\mathbf{I}_{\mathrm{p}}^{\mathrm{N}}$ $\mathrm{N}$ conventional serial coding systems, coding-induced parity-check bits are added to the encoded data sequences so that the system bit rate is inevitably increased. In practical lightwave systems with standard bit rate, change of system bit rate is generally unacceptable. Nevertheless, FEC for synchronous optical network (SONET) was proposed by Grover and Moore [1] wherein they follow conventional serial coding approach but put parity-check bits in unassigned overheads of the SONET frame. System bit rate thereby is unchanged. The results show that the performance of a dispersion-limited lightwave system is improved drastically. However, as parity-check bits are put on some specific overheads, the required signal processing in the encoder/decoder becomes complicated, thus limiting its operating speed to the STS-1 level or STS-3 at the most. In a high-speed system, many encoder/decoder pairs are therefore required. Extending to high-capacity WDM systems with many high-speed wavelength channels, numerous encoders/decoders will be necessary.

Taking advantage of the parallel nature of WDM systems, an interchannel parallel WDM coding scheme was introduced in [2] to overcome the above-mentioned difficulty by putting parity-check bits in additional wavelength channels. The system employs Hamming codes for single-error correction in which the number of parity-check channels $r$ increases with the number of data channels and $r \geq 3$. In this paper we propose a novel coding scheme for high-speed WDM systems based on product codes, that requires only one encoder/decoder and two additional wavelength channels to correct errors in all the wavelength channels. The implementation of product codes in conventional serial transmission is rather difficult since the encoding/decoding involves two kinds of codes in an array form whereas the transmission channel is serial [3]. In contrast, the implementation of the proposed WDM coding system is straightforward and simple since the array codeword can be

Paper approved by L. G. Kazovsky, the Editor for Optical Communications of the IEEE Communications Society. Manuscript received November 15, 1994; revised May 11, 1995.

The authors are with the Department of Communication Engineering and Center for Telecommunications Research, National Chiao-Tung University,

Hsinchu, Taiwan, R.O.C.

Publisher Item Identifier S 0090-6778(96)00808-2. encoded, transmitted and decoded all in parallel, thereby the resulting coding scheme is simple and efficient.

\section{SYSTEM DESCRIPTION}

The architecture of the product-coded WDM coding (PCWDMC) system is shown in Fig. 1. At the transmitting end, $m$ data channels $\left(D_{1}, \cdots, D_{m}\right)$, each having STS-N rate, are fed in parallel to an FEC encoder to produce two parity check channels $\left(P_{C}, P_{R}\right)$. The $m$ data channels are completely unchanged after the encoder and the two parity channels have the same STS-N rate as the data channels. The $m+2$ codeword channels then modulate $m+2$ lasers at different wavelengths to form a WDM transmission. At the receiving end, the wavelength channels are received separately by $m+2$ receivers and then decoded parallelly by an FEC decoder to correct possible errors in the data channels.

In the PC-WDMC system, a simple parity-check code is used for single-error correction. As shown in Fig. 2, each one of the $m$ data channels offers $b$ bits to form an encoded data block of $m b$ bits $\left(d_{11}, \cdots, d_{m b}\right)$. The data block is configured as an $m \times b$ array consisting of $m$ rows and $b$ columns, with the $i$ th row $\left(d_{i 1}, \cdots, d_{i b}\right)$ offered by the $i$ th data channel $\left(D_{i}\right)$. A simple parity-check code is applied to every row and every column: the row parity check (RPC) bits $\left(P_{R 1}, P_{R 2}, \cdots, P_{R m}\right)$ are given by

$$
P_{R i}=d_{i 1} \oplus d_{i 2} \oplus \cdots \oplus d_{i b}, \quad i=1,2, \cdots, m
$$

and the column parity check (CPC) bits $\left(P_{C 1}, P_{C 2}\right.$, $\left.\cdots, P_{C m}\right)$ are obtained as

$$
P_{C j}=d_{1 j} \oplus d_{2 j} \oplus \cdots \oplus d_{m j}, \quad j=1,2, \cdots, b
$$

where the symbol " $\oplus$ " denotes binary addition. Here even parity is employed.

As shown in Fig. 3, the row and column parity bits are transmitted separately by the row parity channel $\left(P_{R}\right)$ and the column parity channel $\left(P_{C}\right)$, respectively. The $m$ data channels and the two parity channels all have the same transmission rate. The column parity channel is occupied completely by the CPC bits since the number of column parity bits in a codeword is the same as the number of data bits in each data channel. In the row parity channel, in addition to transmitting the RPC bits, a frame alignment word (FAW) is needed, to be used at the receiving end, to distinguish an encoded block from its preceding and succeeding blocks. Owing to the FAW, the frame length $b$ must be greater than the number of data channels $m$.

In the PC-WDMC system the input channels of the FEC encoder/decoder should be synchronized so that they can be 


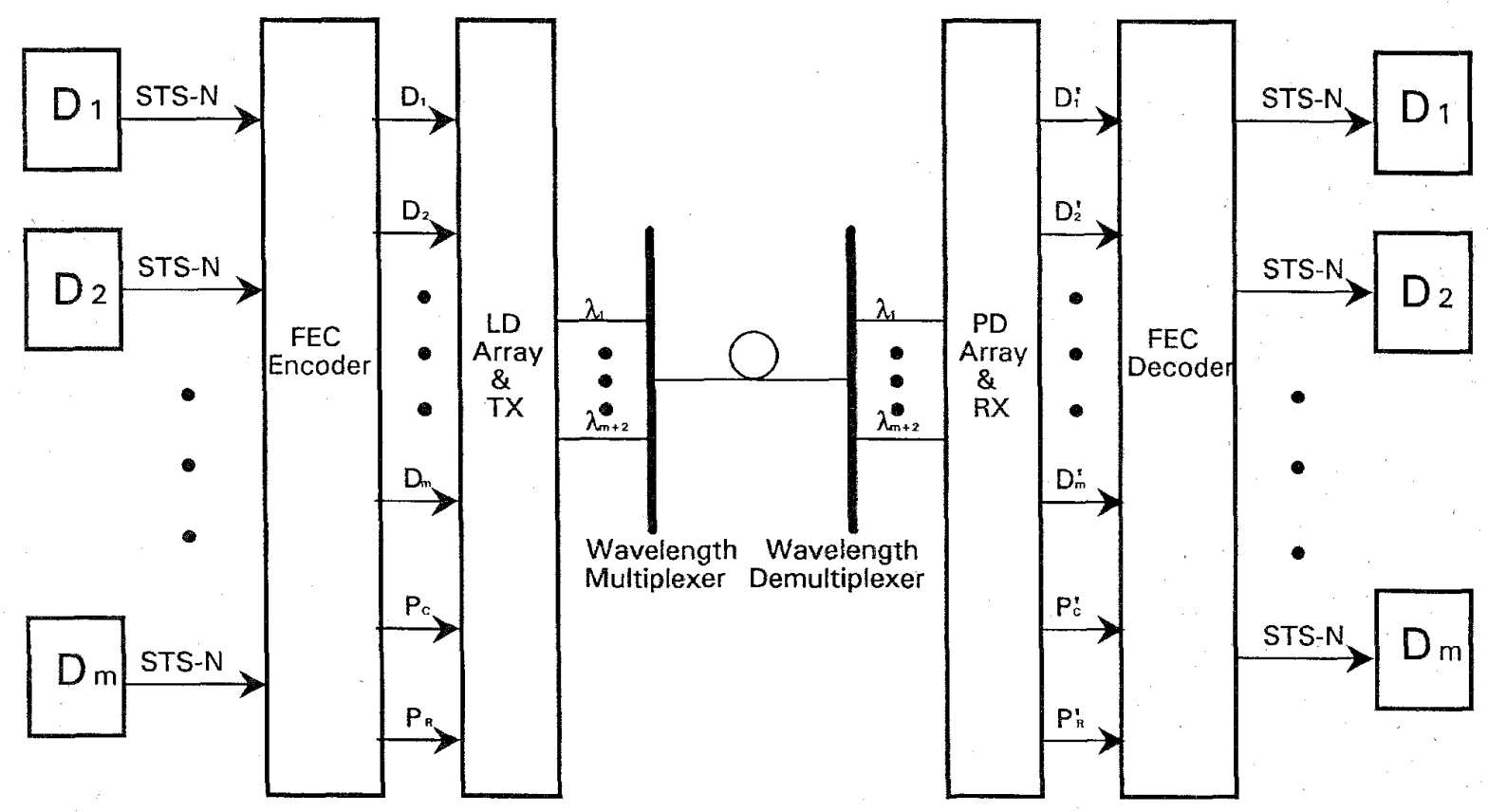

Fig. 1. Block diagram of the PC-WDMC system. $D_{1}, \cdots, D_{m}$ are data channels and $\left(P_{C}, P_{R}\right)$ are parity channels.

RPC

\begin{tabular}{|c|c|c|c|c|c|c|}
\hline$P_{\mathrm{R} 1}$ & $d_{11}$ & $\mathrm{~d}_{12}$ & $\bullet$ & $\bullet$ & • & $d_{1 b}$ \\
\hline$P_{R 2}$ & $d_{21}$ & $d_{22}$ & $\bullet$ & - & • & $\mathrm{d}_{2 \mathrm{~b}}$ \\
\hline $\begin{array}{l}\bullet \\
\bullet \\
\bullet\end{array}$ & $\begin{array}{l}\cdot \\
\bullet \\
\bullet\end{array}$ & $\begin{array}{l}\bullet \\
\bullet \\
\bullet\end{array}$ & $\bullet$ & $\bullet$ & $\bullet$ & $\begin{array}{l}\bullet \\
\bullet \\
\bullet\end{array}$ \\
\hline $\mathrm{P}_{\mathrm{Rm}}$ & $d_{m 1}$ & $\mathrm{dm}$ & $\bullet$ & $\bullet$ & $\bullet$ & $\mathrm{dmb}$ \\
\hline & $\mathrm{P}_{\mathrm{C} 1}$ & $\mathrm{P}_{\mathrm{C} 2}$ & $\bullet$ & $\bullet$ & $\bullet$ & $\mathrm{P}_{\mathrm{cb}}$ \\
\hline
\end{tabular}

Fig. 2. Frame structure of the PC-WDMC system. Each row has $b$ bits and each column has $m$ bits. Row and column parity bits are produced in each row and each column.

encoded/decoded simultaneously. As the incoming data channels to the FEC encoder may come from different locations, they may not be synchronized. Also the clock instabilities in SONET networks may cause a group of synchronized data channels losing synchronism during a short time period. Since the PC-WDMC system is independent of the frame formats of the data channels, the synchronization problem can be overcome by using buffers before the encoder to phase align the incoming data bits. On the other hand, owing to group velocity mismatch among the wavelength channels, the $m+2$ channel data in general will not reach the receiving end simultaneously, leading to the bit-skew problem [4]. There are two possible means to compensate bit-skew at the receiving end: 1) the optical compensation scheme uses fiber delay lines after the WDM demultiplex to compensate different propagation delay of the wavelength channels; 2) the electrical compensation scheme uses the slowest wavelength channel as

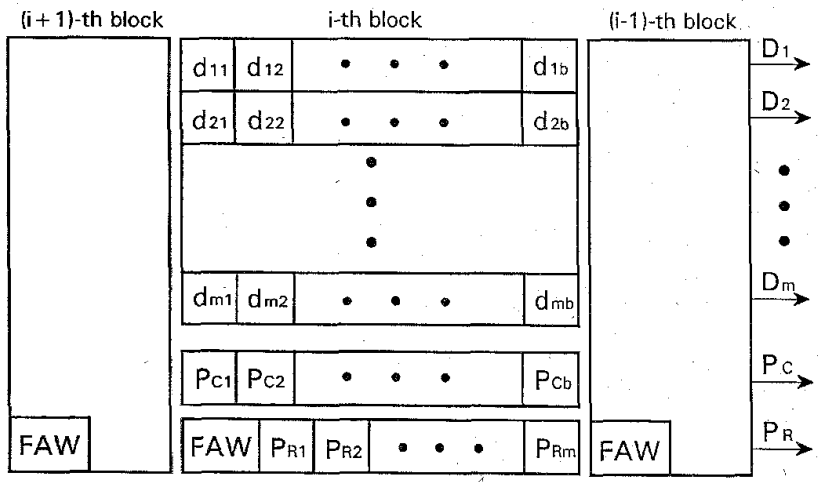

Fig. 3. Transmission of the PC.WDMC system. Column parity bits are transmitted by the $P_{C}$ channel, row parity bits and a frame alignment word are transmitted by the $P_{R}$ channel. The FAW is used to distinguish a frame from its preceding and succeeding frames.

the trigger signal to load all the channels to a buffer thereby achieving synchronization at the buffer output [2].

At the decoder, a simple parity check is executed for every row and every column. The check bits for row and column are obtained as

$$
\begin{aligned}
& X_{R i}=d_{i 1}^{\prime} \oplus d_{i 2}^{\prime} \oplus \cdots \oplus d_{i b}^{\prime} \oplus P_{R i}^{\prime}, \quad i=1,2, \cdots, m \\
& X_{C j}=d_{1 j}^{\prime} \oplus d_{2 j}^{\prime} \oplus \cdots \oplus d_{m j}^{\prime} \oplus P_{C j}^{\prime}, \quad j=1,2, \cdots, b
\end{aligned}
$$

where $d_{i j}^{\prime}$ and $P_{R, C i}^{\prime}$ are the received data bits and row/column parity bits, respectively.

The error correcting procedure is simple. If there is no error in the received codeword, all check bits will be " 0. " In this case, the decoder just outputs the received data sequences without any correction. If one error occurs, e.g., $d_{i j}^{\prime}=\bar{d}_{i j}$, then $X_{R i}=X_{C j}=1$ and all the other check bits remain 


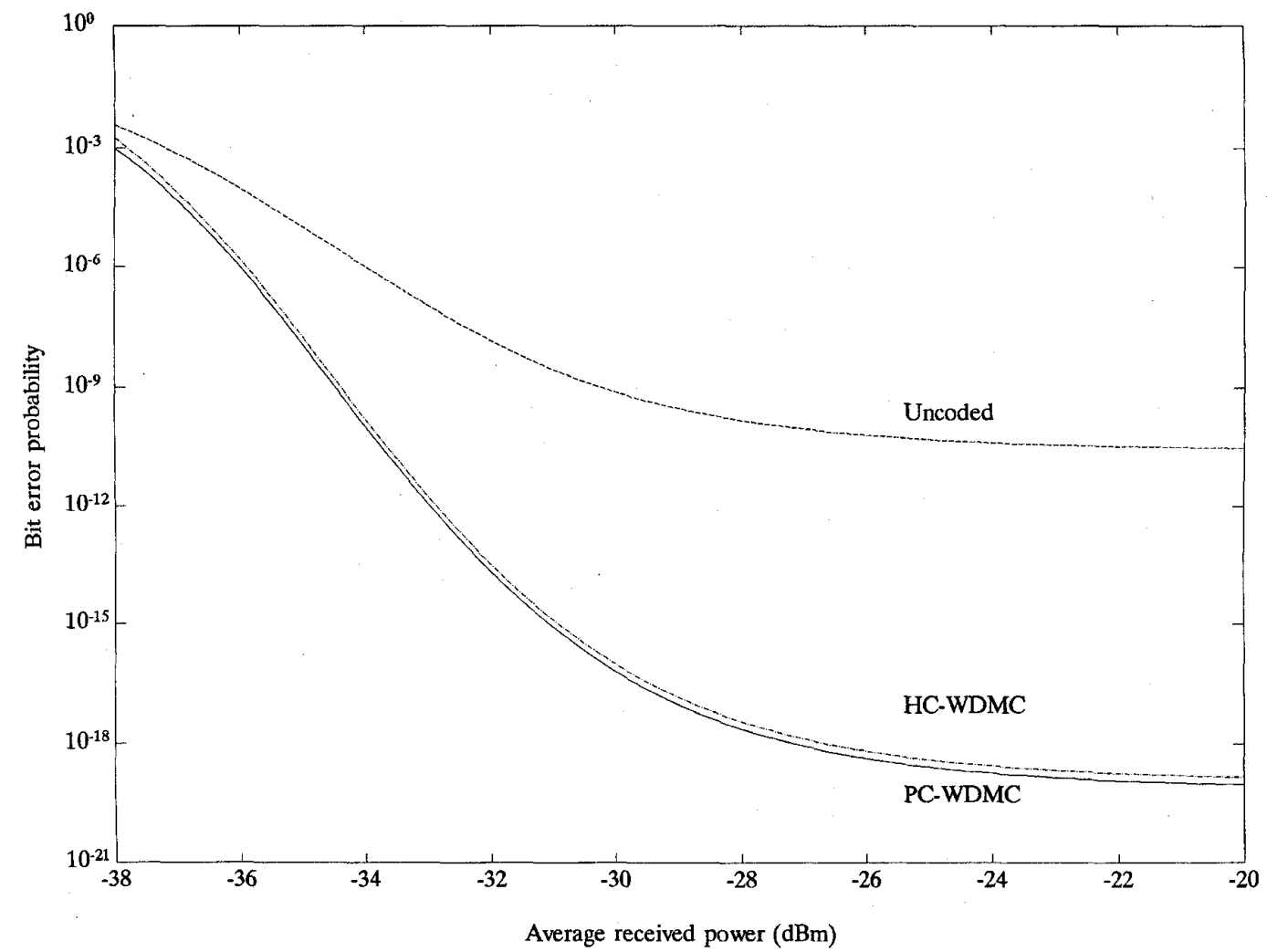

Fig. 4. BER as a function of received signal power for a lightwave system. The parameters used in the calculation are the same as those in [5] and the error floor is caused mainly by reflection noise. Here we assume the PC-WDMC system and the Hamming coded WDM coding (HC-WDMC) system have the same codeword length $(n=100)$.

" 0 ," and thus the error bit can be decided and then corrected exactly by the decoder.

The simple decoder can correct single error only. For more than one error, the correctable errors depend on error patterns and miscorrection may happen [3]. For example, if two errors occur in the same row or column of the array codeword, they cannot be detected and therefore cannot be corrected. If they occur at different row and column, they can be detected and corrected; but the decoder will miscorrect two other bits also.

Since the simple single-error-correcting scheme is able to decline the error rate from $O\left(P_{e}\right)$ to $O\left(P_{e}^{2}\right)$, it is enough to improve the performance of low BER lightwave system significantly. And because of its simplicity, it is suitable for high-speed lightwave systems.

\section{Performance Evaluation}

The word error probability $P_{w}$ after error correction is

$$
\begin{aligned}
P_{w} & =\sum_{i=2}^{n} C_{i}^{n} P_{e}^{i}\left(1-P_{e}\right)^{n-i} \\
C_{i}^{n} & =\frac{n !}{(n-i) ! i !}
\end{aligned}
$$

where $n(=m b+m+b)$ is the codeword length and $P_{e}$ is the BER before decoding. Here we take $P_{e}$ as the worst BER among the $m+2$ wavelength channels to obtain a conservative estimation. The bit error probability for a data channel can be derived from the word error probability, given by

$$
P_{e, F E C}=\frac{1}{m b} \sum_{i=2}^{n} E(i) C_{i}^{n} P_{e}^{i}\left(1-P_{e}\right)^{n-i}
$$

where $E(i)$ is the mean number of error bits within the $m b$ data bits after error correction given $i$ primary error bits. For a low BER system, it is easy to conclude that $P_{e, F E C}$ is dominated by $i=2$. Thus we have

$$
P_{e, F E C} \simeq \frac{1}{m b} E(2) C_{2}^{n} P_{e}^{2}\left(1-P_{e}\right)^{n-2} .
$$

Consider the case that two errors occur in a codeword. The errors may occur in either the same bit stream or different channels, and depending on different error patterns, they may result in 0,1 , or 2 decoded errors. All the possible patterns, their occurrence probabilities, and the resultant decoded errors had been considered to obtain the parameter $E(2)$, given as

$$
E(2)=\frac{m b(m b+m+b)}{C_{2}^{n}} .
$$

Thus

$$
\begin{aligned}
P_{e, F E C} & \simeq(m b+m+b) P_{e}^{2}\left(1-P_{e}\right)^{n-2} \\
& =n P_{e}^{2}\left(1-P_{e}\right)^{n-2} .
\end{aligned}
$$


To demonstrate the effectiveness of WDM coding in reducing the BER floor, we plot in Fig. 4 the uncoded and the decoded BER for a lightwave system. Here the noises under consideration are: circuit noise, shot noise, laser intensity noise, reflection-induced noise, mode-partion noise, and chirp noise. The analysis method follows that in [5] with practical parameters. The figure indicates that the PC-WDMC system has almost the same performance as the Hamming coded WDM coding system proposed in [2] and can decline the BER floor significantly. In terms of coding gain, the PC-WDMC system has approximately $4 \mathrm{~dB}$ coding gain at $B E R=$ $10^{-9}$, and the coding gain becomes infinite if the desired BER is below the BER floor. Also notice that the coding is applied to the $m$ data channels simultaneously just with one encoder/decoder. In the example the error fioor is caused mainly by reflection noise. However, the WDM coding is applicable to decline the error floor of dispersion-limited systems also.

\section{CONCLUSIONS}

As the encoding/decoding is complicated and the number of encoder/decoder increases with the number of data channels and system bit rate, conventional serial coding is not a very good approach for high-speed WDM systems. Here we propose a product-coded WDM coding system, that is completely different from conventional serial coding systems. The PCWDMC system fits exactly the parallel nature of WDM transmission, that encodes, transmits and decodes the array codeword all in parallel. The system has many advantages: 1) simple encoder/decoder circuit, 2) only one encoder/decoder to correct errors in all the data channels, and 3) just needs two additional wavelength channels.

\section{REFERENCES}

[1] W. D. Grover and T. E. Moore, "Design and characterization of an error correcting code for the SONET STS-1 tributary," IEEE Trans. Commun. vol. 38 , no. 4 , pp. 467-476, Apr. 1990.

[2] S. K. Shao and M. S. Kao, "WDM coding for high-capacity lightwave systerns," J. Lightwave Technol., vol. 12, no. 1, pp. 137-148, Jan. 1994.

[3] S. Lin and D. J. Costello Jr., Error Control Coding: Fundamental and Applications. Englewood Cliffs, NJ: Prentice-Hail, 1983.
[4] M. L. Loeb and G. R. Stilwell, "High-speed data transmission on an optical fiber using a byte-wide WDM system," J. Lightwave Technol., vol. 6, no. 8, pp. 1306-1311, Aug. 1988.

[5] T.M. Shen and G. P. Agrawal, "Computer simulation and noise analysis of the system performance of $1.55 \mu \mathrm{m}$ single-frequency semiconductor lasers," J. Lightwave Technol., vol. LT-5, no. 5, pp. 653-659, May 1987.

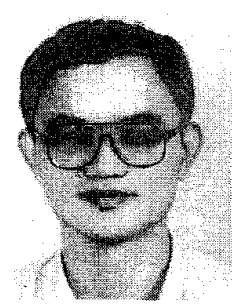

Ming-Seng Kao (''89-M'90) was born in Taipei, Taiwan, R.O.C., in 1959. He received the B.S.E.E. degree from the National Taiwan University in 1982, the M.S. degree in optoelectronics from the National Chiao-Tung University, Hsinchu, Taiwan, in 1986, and the Ph.D. degree in electrical engineering from the National Taiwan University in 1990.

From 1986 to 1987 he was an Assistant Researcher at the Telecommunications Laboratories, Chung-Li, Taiwan. In 1990, he joined the faculty of National Chiao-Tung University, Hsinchu, Taiwan, where he is now a Professor in the Communications Engineering Department. Between 1993 and 1994, he was a Visiting Professor at the Swiss. Federal Institute of Technology (ETH), Zurich, Switzerland, where he worked in the area of optical communications. He is currently interested in high-speed optical networks and CATV networks.

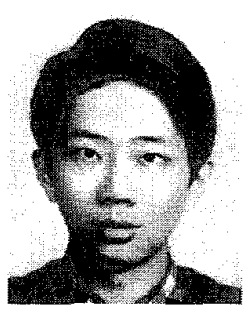

Hsin-Yuan Chen was born in Taipei, Taiwan, R.O.C., in 1969. He received the B.S. and M.S. degrees in communication engineering from the National Chaio-Tung University, Hsinchu, Taiwan, in 1992 and 1994, respectively.

He had been involved in the research of lightwave communications and holds the electrical and electronic licenses. His interests include optical fiber systems and digital communications.

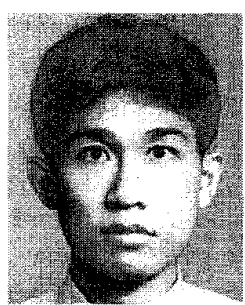

Das Lin was born in Taiwan, R.O.C., in 1969. He received the B.S. degree in control engineering and the M.S. degree in communication engineering, both from the National Chiao-Tung University, Hsinchu, Taiwan, in 1992 and 1994, respectively.

$\mathrm{He}$ is currently serving in the Army as a second lieutenant. His interests include optical fiber systems, coding and networks. 